\title{
Another Issue for the Modern Vascular Specialist to Consider: Advanced Imaging Software
}

Journal of Endovascular Therapy 2019, Vol. 26(5) 688-690

(C) The Author(s) 2019

Article reuse guidelines:

sagepub.com/journals-permissions DOI: $10.1177 / 1526602819866437$

www.jevt.org

@SAGE

\section{Maurizio Domanin, $M D^{1,2}$ and Santi Trimarchi, $M D, P^{1,2}$}

\section{Keywords}

aorta, aortic sizing, complications, computed tomography angiography, endograft, endovascular aortic repair, imaging, intervention planning, medical software, stent-graft, surveillance

Since the advent of the endovascular era for abdominal and thoracic aortic lesion repair, vascular specialists have experienced an outstanding transformation of our work, which altered not only our surgical practice but added radiological imaging visualization for aortic sizing, intervention planning, and surveillance programs. We have moved from the study of computed tomography angiography (CTA) images printed on the old $\mathrm{x}$-ray films and sizing vessels with paper rules or compasses to the use of Digital Imaging and Communications in Medicine (DICOM) files visualized with advanced software. This has allowed us to easily obtain 3-dimensional reconstructions, maximum intensity projections, and multiplanar reformations in a Picture Archiving and Communication System application available on our laptops and smart phones.

Despite all these advances, some imaging evaluations during follow-up remain frankly "difficult" to interpret. Who has not had a little bit of anxiety, more common with very complex cases, watching not particularly encouraging postoperative CTA scans? Who has not suspected after viewing initially successful postoperative images that these results could be only temporary? Such feelings are more frequent after we treat aortic patients presenting with short, tortuous, and calcified necks, basically those patients who are totally outside the instructions for use and who may represent up to $80 \%$ of our patient volume. ${ }^{1-3}$

Currently, imaging software is widely available to drill down the CTA scans and to determine apposition surface in the proximal and distal landing zones, apposition of the endograft fabric with the aortic wall, shortest apposition length, endograft inflow and outflow diameters, and distances between fabric extremes and anatomical landmarks. In addition, these tools allow us to define the stability of endograft position, supporting our practice by offering an essential diagnostic aid in endovascular aortic repair surveillance. $^{4}$

Despite tremendous technological refinements in endografts, the fact is that life-threatening complications, such as migration, fractures, and endoleaks, still occur during longer follow-up, representing the most common causes of reintervention. Sometimes the endograft seems to be an "idol with feet of clay" as a result of its inability to achieve stable aortic sealing. Looking at the big picture, long-term instability of aortic endografts, both abdominal and thoracic, remains a concern in up to one-fourth of cases. ${ }^{3,5,6}$ Such issues make lifelong surveillance mandatory, a point that has become more important since the management trends for aortic pathologies have expanded the treatment to younger patients with longer life expectancies, in addition to octo- and nonagenarians. ${ }^{1}$

The complexity of thoracic aortic diseases, their inaccessibility to ultrasound examination, and the extreme variability of postoperative complications compel us to submit our patients to CTA or magnetic resonance angiography at 30 days, 6 months, and then yearly for the rest of their lives. ${ }^{2,7}$ Such protocols unfortunately need to include major concerns such as radiation exposure (only for CTA), contrast-induced nephropathy, and adverse reactions to contrast agents. ${ }^{8}$

In the October 2019 issue of the JEVT, van Noort and colleagues ${ }^{4}$ report the validation of the Vascular Image Analysis software, which might one day be helpful clinically for semiautomatic determination of minimal spatial differences (in the order of $0.1 \mathrm{~mm}$ ) in radiological images, far beyond the capabilities of the human eye. The next evolution would be to develop completely automatic software to overcome the human interface and inter-/intraobserver bias and thus achieve greater objectivity in clinical measurements.

'Department of Clinical and Community Sciences, University of Milan, Italy

${ }^{2}$ Unit of Vascular Surgery, Fondazione IRCCS Cà Granda Ospedale Maggiore Policlinico, Milan, Italy

Invited commentaries published in the Journal of Endovascular Therapy reflect the opinions of the author(s) and do not necessarily represent the views of the Journal, the INTERNATIONAL SOCIETY OF ENDOVASCULAR SPECIALISTS, or SAGE Publications Inc.

\section{Corresponding Author:}

Santi Trimarchi, Department of Clinical and Community Sciences, University of Milan, IRCCS Fondazione Cà Granda Ospedale Maggiore Policlinico, Via Francesco Sforza 35, 20122 Milan, Italy.

Email: santi.trimarchi@unimi.it 


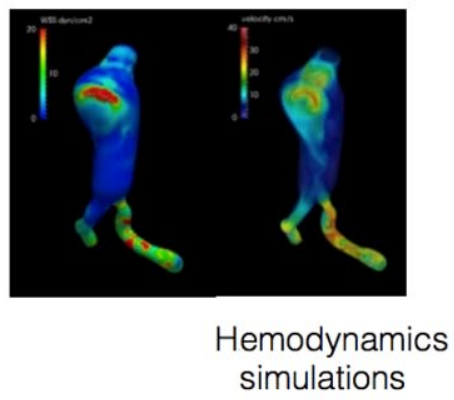

Repair device AUTOMATIC PLANNER

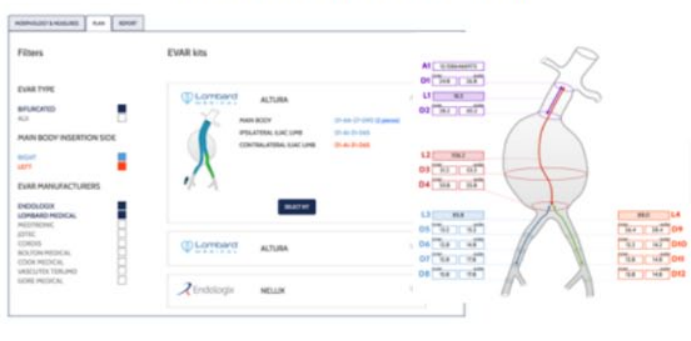

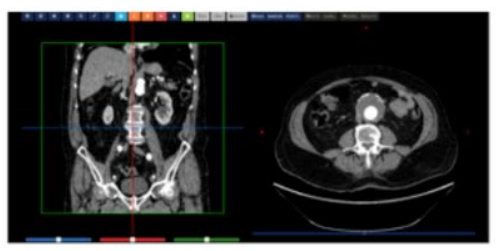

DICOM images 3D exploration

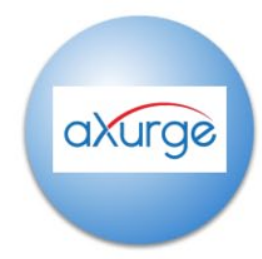

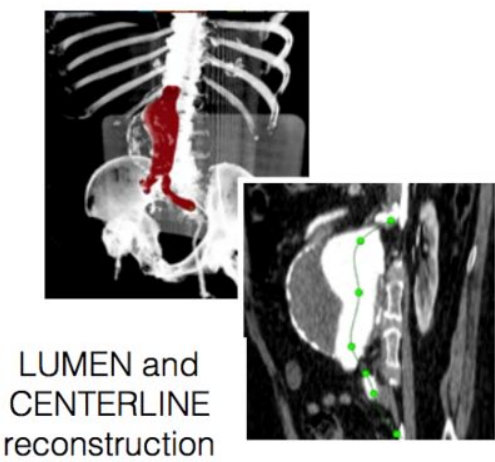

MPR exploration

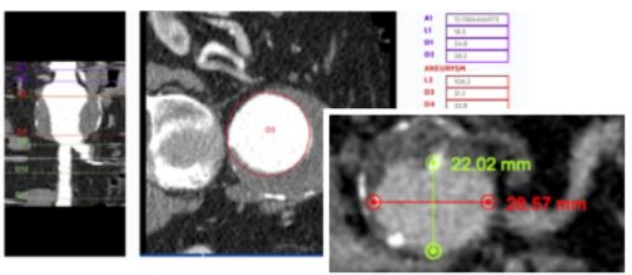

Figure I. Example of the aXurge project functions. DICOM, Digital Imaging and Communications in Medicine; MPR, multiplanar reformat.

Migration of the endograft is a very complex issue involving several anatomical factors. On this background, drag forces in thoracic endografts, that is, the resistance forces caused by blood flow against the body of the graft, have been studied and measured. ${ }^{9}$ Drag forces are predominantly directed sideways in the abdominal aorta and upward in the proximal thoracic aorta, ${ }^{10,11}$ where the magnitude and direction are affected by the curvature and tortuosity. ${ }^{12}$ Similarly, higher drag forces have been detected in patients with a descending thoracic aortic aneurysm presenting with a high degree of tortuosity, a potential cause of endograft migration. ${ }^{13}$ The increased drag forces have been investigated using computational fluid dynamics (CFD) simulations in the proximal and distal descending aorta. ${ }^{12,13}$

With aging, the aortic wall deteriorates and become larger, longer, and more tortuous. These features are more common for pathological aorta and can create problems for stent-graft mechanical stability, favoring device migration. In addition, other factors that could influence the magnitude of drag forces appear to be blood pressure control during follow-up, heart rate, blood viscosity, and potentially aortic stiffness and its related pulse waveform.
The time is rapidly coming when new advanced software will tremendously help the modern vascular specialist manage complex aortic cases. At the same time, technological applications will be able to store radiological images, analyze anatomical features, size and plan endovascular procedures, perform aneurysm segmentation in real time, run CFD simulations, and execute analyses to evaluate the stability of aortic devices and suggest long-term prognosis. One example among several is the upcoming aXurge program (www.axurge.com; Figure 1). The diffusion of new medical software will be aided by other new technologies, such as high-performance computers and the evolution of mobile wireless systems.

\section{Declaration of Conflicting Interests}

The author(s) declared no potential conflicts of interest with respect to the research, authorship, and/or publication of this article.

\section{References}

1. Wong S, Mastracci TM, Katsargyris A, et al. The role of mandatory lifelong annual surveillance after thoracic endovascular repair. J Vasc Surg. 2012;56:1786-1793. 
2. Lau C, Feldman DN, Girardi LN, et al. Imaging for surveillance and operative management for endovascular aortic aneurysm repairs. $J$ Thorac Dis. 2017;9(suppl 4):S309-S316.

3. Giles KA, Beck AW, Lala S, et al. Implications of secondary aortic intervention after thoracic endovascular aortic repair for acute and chronic type B dissection. J Vasc Surg. 2019;69:1367-1378.

4. van Noort K, Schuurmann RCL, Post Hospers G, et al. A new methodology to determine apposition, dilatation, and position of endografts in the descending thoracic aorta after thoracic endovascular aortic repair. J Endovasc Ther. 2019;26(5):679687.

5. Conway AM, Qato K, Mondry LR, et al. Outcomes of thoracic endovascular aortic repair for chronic aortic dissections. J Vasc Surg. 2018;67:1345-1352.

6. Pellenc Q, Girault A, Roussel A, et al. Regarding "Outcomes of thoracic endovascular aortic repair for chronic aortic dissections. J Vasc Surg. 2018;67:1942-1943.

7. Zierler RE, Jordan WD, Lal BK, et al. The Society for Vascular Surgery practice guidelines on follow-up after vascular surgery arterial procedures. J Vasc Surg. 2018;68: 256-284.
8. Kumamaru KK, Hoppel BE, Mather RT, et al. CT angiography: current technology and clinical use. Radiol Clin North Am. 2010;48:213-217.

9. Figueroa CA, Taylor CA, Yeh V, et al. Effect of curvature on displacement forces acting on aortic endografts: a 3-dimensional computational analysis. J Endovasc Ther. 2009;16: 284-294.

10. Prasad A, To LK, Gorrepati ML, et al. Computational analysis of stresses acting on intermodular junctions in thoracic aortic endografts. J Endovasc Ther. 2011;18:559-568.

11. Figueroa CA, Taylor CA, Chiou AJ, et al. Magnitude and direction of pulsatile displacement forces acting on thoracic aortic endografts. J Endovasc Ther. 2009;16:350-358.

12. Marrocco-Trischitta MM, van Bakel TM, Romarowski $\mathrm{RM}$, et al. The modified arch landing areas nomenclature (MALAN) improves prediction of stent graft drag forces: proof of concept by computational fluid dynamics modelling. Eur J Vasc Endovasc Surg. 2018;55:584-592.

13. Belvroy VM, Romarovski RR, Van Herwaarden JA, et al. Computational fluid dynamics in descending thoracic aortic aneurysm: tortuosity associated with displacement forces [abstract]. J Vasc Surg. 2019;69:e34. 